\title{
Temperature- and size-scaling of phytoplankton population growth rates: Reconciling the Eppley curve and the metabolic theory of ecology
}

\author{
Colin T. Kremer, ${ }^{1,2, a *}$ Mridul K. Thomas (D), ${ }^{3}$ Elena Litchman ${ }^{1,4}$ \\ ${ }^{1}$ W. K. Kellogg Biological Station, Michigan State University, Hickory Corners, Michigan \\ ${ }^{2}$ Department of Plant Biology, Michigan State University, East Lansing, Michigan \\ ${ }^{3}$ Department of Aquatic Ecology, Eawag: Swiss Federal Institute of Aquatic Science and Technology, Dübendorf, Switzerland \\ ${ }^{4}$ Department of Integrative Biology, Michigan State University, East Lansing, Michigan
}

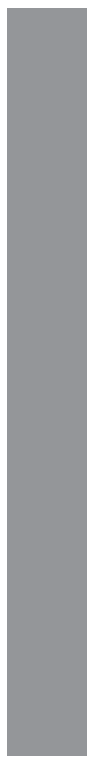

\begin{abstract}
Quantifying how environmental factors control the growth of phytoplankton communities is essential for building a mechanistic understanding of global biogeochemical cycles and aquatic food web dynamics. The strong effects of temperature on population growth rate have inspired two frameworks-the Eppley curve and the metabolic theory of ecology_that produce different quantitative relationships and employ distinct statistical approaches. Reconciling these relationships is necessary to ensure the accuracy of ecosystem models. In this paper, we develop ways to compare these frameworks, overcoming their methodological differences. Then, analyzing an extensive dataset ( $>4200$ growth rate measurements), we find that increases in population growth rate with temperature are consistent with metabolic theory, and weaker than previous estimates of the Eppley curve. A $10^{\circ} \mathrm{C}$ temperature increase will increase growth rates by a factor of 1.53 , rather than 1.88 as in previous studies of the Eppley curve. Size and functional group membership are also critical. Population growth rates decrease with size, but much less strongly that metabolic theory predicts. The growth rates of different functional groups scale similarly with temperature, but some groups grow faster than others, independent of temperature. Our results reconcile the analytical methods of the Eppley curve and metabolic theory, demonstrate that metabolic theory's temperature-scaling predictions are more accurate, and provide new insights into the factors controlling phytoplankton growth. To avoid over-estimating the effects of temperature on primary productivity, the parameterization of ecosystem models should be revised.
\end{abstract}

Phytoplankton communities regulate global biogeochemical cycles and support most aquatic food webs. It is critically important to understand the factors that control their growth, influencing current and future primary productivity. Temperature is one such factor, strongly affecting the growth of phytoplankton populations, mediated by its effects on the rate of biochemical reactions. Temperature effects are the primary focus of this paper; however, population growth rates (hereafter, simply "growth rate") also depend on cell size: phytoplankton species with larger cells typically grow

*Correspondence: colin.kremer@yale.edu

apresent address: Department of Ecology and Evolutionary Biology, Yale University, New Haven, Connecticut

Additional Supporting Information may be found in the online version of this article. more slowly than those with smaller cells. Relationships between size, temperature, and growth rate have been documented for many years, in both the lab and the field, but the precise representation of these relationships has been disputed. The dominant bodies of thought, or frameworks, addressing the temperature-dependence of population growth are the highly influential Eppley curve (Eppley 1972) and the metabolic theory of ecology, which we abbreviate as "MTE" (Gillooly et al. 2001; Brown et al. 2004). The two frameworks have historically provided quantitatively different estimates of the strength of temperature-dependence (Table 1). Both approaches also differ in their equations, statistical methods, and foundations: the Eppley curve is based on characterizations of an empirical relationship, while MTE provides theoretical predictions emerging from first principles. These differences have inhibited rigorous comparisons of the two frameworks. In this paper, we reconcile the 
Table 1. Comparing the predictions of the Eppley and MTE approaches to understanding the effects of temperature on growth.

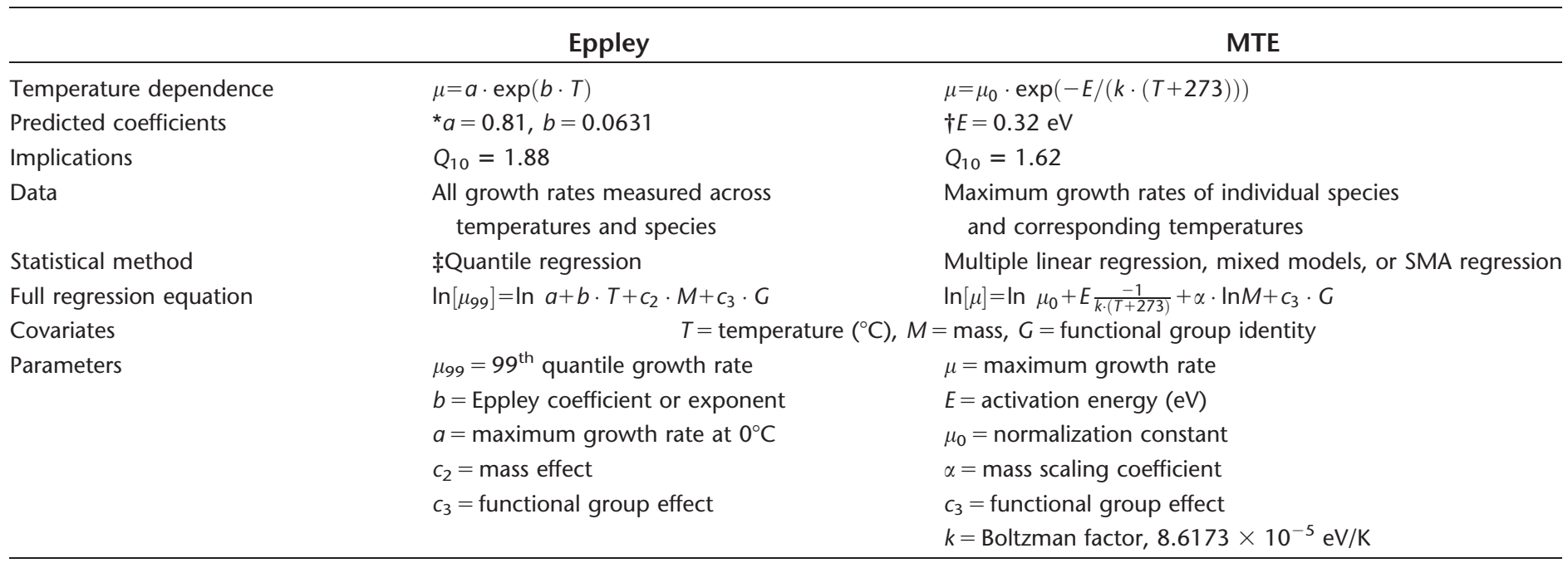

* Bissinger et al. (2008), Eppley (1966).

† Savage et al. (2004a), Allen et al. (2005).

‡ Koenker (2005), Cade and Noon (2003).

contrasting Eppley curve and MTE relationships, making use of an extensive compilation of growth rate data. This represents a critical step in developing a mechanistic, quantitatively accurate, and predictive understanding of phytoplankton communities and marine ecosystems at large.

An exponential relationship between maximum growth rate of phytoplankton and temperature was reported first by Eppley (1972). Since this seminal paper, the relationship Eppley estimated has become a fundamental component of our understanding of how primary productivity is constrained by temperature. For example, the "Eppley curve" is used in algorithms estimating primary productivity from satellite observations (Morel 1991; Antoine et al. 1996; and modified in Behrenfeld and Falkowski 1997), and implemented in a wide range of marine ecosystem models (select examples include Tett et al. 1985; Doney et al. 1996; Palmer and Totterdell 2001; Taucher and Oschlies 2011; Thomas et al. 2012; Stock et al. 2014). Together, the data derived from satellite observations and the predictions made by ecosystem models are essential to studying aquatic ecology, including detecting and predicting the effects of climate change on primary productivity. Thus, the Eppley curve's accuracy is vital, including both its parameters and the ideas it embodies. Mathematically, the Eppley curve can be written as:

$$
\mu=a \exp (b \cdot T)
$$

In this equation, $\mu$ is the maximum growth rate of any phytoplankton species, which varies with temperature, T. Parameter $a$ controls the height of the exponential function at $0^{\circ} \mathrm{C}$ while $b$ determines how strongly $\mu$ rises with temperature, a value often referred to as the Eppley exponent. Eppley's initial estimates of these parameters $(a=0.59$ and $b=0.0633)$ have been updated using more data and rigorous quantile regression methods $(a=0.81$ and $b=0.0613)$, the values we use throughout the rest of this paper (Table 1; Bissinger et al. 2008; but see also Brush et al. 2002). Ultimately, the Eppley curve is a phenomenological description of the temperaturedependence of growth, whose parameters are estimated from data rather than predicted from first principles.

Metabolic theory (MTE) offers a contrasting, mechanistic description of the same empirical pattern, derived from studying the factors constraining individual metabolic rates (Gillooly et al. 2001; Brown et al. 2004). It focuses specifically on the biochemical reactions essential for life, which depend on temperature and the supply of substrate(s). Assuming substrates are not limiting, reaction rates will depend only on temperature, and can be described by the Arrhenius-van't Hoff equation: $k \propto e^{-E / R T}$. This states that the rate constant of a reaction, $k$, is proportional to an exponential function of: temperature, $T$, the Boltzmann constant $(R=8.617 \times$ $10^{-5} \mathrm{eV} \mathrm{K}^{-1}$ ), and the activation energy of the reaction, $E$. Conceptually, $E$ describes the energy required to initiate a specific chemical reaction and determines the sensitivity of $k$ to changes in temperature. MTE further assumes that the rate of the slowest chemical reaction (lowest $E$ ) that is essential for life will determine just how quickly individuals and populations can grow, such that $\mu \propto k$ (Savage et al. 2004a). As such, $E$ is analogous to the Eppley exponent $b$, in that they both control the sensitivity of maximum growth rate to temperature. Despite this, it is impossible to directly compare these coefficients because the Eppley curve is a function 
of $T$, while the Arrhenius-van't Hoff equation depends on $-1 / T$. Fortunately, as we will see, an approximation enables us to compare these competing frameworks (Gillooly et al. 2002; Supporting Information: Converting between MTE and Eppley).

MTE makes explicit predictions for the value of $E$, depending on which biochemical process is rate-limiting for growth. In heterotrophs, this is respiration (ATP synthesis), which has an activation energy of $0.65 \mathrm{eV}$ (Allen et al. 2005; López-Urrutia et al. 2006). Photoautotrophs, such as phytoplankton, are limited instead by photosynthesis, with the lower activation energy of $0.32 \mathrm{eV}$ (Allen et al. 2005; LópezUrrutia et al. 2006). This provides an explicit prediction of the temperature-dependence of the maximum growth rate of phytoplankton, given saturating light and nutrients. Relationships between maximum growth rate and temperature have been widely reported (see meta-analysis of Angilletta et al. 2010), although many of the studies examined report variation from the theoretical predictions of Savage et al. (2004a). There is evidence in plankton that the population growth rate of herbivorous and bacterivorous protists and copepods scales more strongly with temperature than phototrophic protists (Rose and Caron 2007), supporting the general idea that heterotrophs are more sensitive to temperature than autotrophs.

Unlike the Eppley curve, MTE also accounts for the effects of the size of organisms on their metabolic rate and ability to grow. Just as the effect of temperature on individual metabolic rate can be scaled up to provide insights on population growth rate, the effects of an individual's size on its metabolic rate can be propagated similarly (Savage et al. 2004a). Specifically, the maximum growth rate, $\mu_{\max }$, of a population at a fixed temperature is related to the average mass of individuals in the population, $M$, following the relationship $\mu_{\max } \propto$ $M^{\alpha}$ (Savage et al. 2004a). In this expression, $\alpha$ governs the strength of the mass scaling relationship. It has a theoretically predicted value of $-1 / 4$, based on properties related to the optimization of resource uptake and distribution networks (West et al. 1997, 1999a,b; Banavar et al. 1999; Brown et al. 2004; Savage et al. 2004). This implies that populations consisting of larger individuals will grow more slowly than those comprised of smaller individuals (or cells, in the case of phytoplankton). Overall, this predicted relationship has been upheld for organisms ranging from vertebrates to unicellular eukaryotes (Savage et al. 2004a).

However, investigations of size-scaling relationships in phytoplankton tend to reveal lower values of $\alpha$ than MTE predicts. Exact estimates vary depending on details including the taxonomic composition of communities, the metric of size examined (volume vs. mass in carbon), and the location (lab or field) and experimental methods of studies (LópezUrrutia et al. 2006; Marañón 2008; Chen and Liu 2010, 2011; Sal \& López-Urrutia 2011; Sal et al. 2015). As phytoplankton sizes span more than seven orders of magnitude (biovolume in $\mu \mathrm{m}^{3}$, Finkel et al. 2009), even a weak relationship would result in substantial predicted differences in growth rate between large and small species. Aside from identifying the value of $\alpha$, several studies suggest that the relationship between size and growth rate may actually be unimodal, rather than linear (Bec et al. 2008; Chen and Liu 2010; Marañón et al. 2013; Marañón 2015). These patterns may be driven by differences in evolutionary history among species (Sal et al. 2015), although other evidence suggests unimodal relationships occur within taxonomic groups (Raven 1994). While the precise nature of the effects of cell size on phytoplankton growth rate remains a topic of debate, the overall existence of a relationship is not in question. As a result, it is important to jointly consider the effects of size and temperature when studying the factors limiting population growth.

A final consideration is the extreme diversity of the phytoplankton, consisting of groups such as cyanobacteria and diatoms whose evolutionary histories diverged long ago. Many recent studies have found that the relatedness and functional group identity of species is an important predictor of their physiology, ranging from light and nutrient uptake traits (Litchman et al. 2007; Edwards et al. 2012, 2015), to thermal tolerance traits (Thomas et al. 2016). Similar taxonomic effects have been found in the metabolic theory literature across wide swaths of life. For example, groups from unicellular organisms to fish and amphibians have identical slopes relating their mass-corrected metabolic rate to temperature, but their normalization constants (intercepts) are quite different (Gillooly et al. 2001; Brown et al. 2004). At best, ignoring these effects may obscure relationships across species, while in some cases the inferred shape of relationships may change entirely (e.g., Bickel et al. 1975; Clark et al. 2011; Sal et al. 2015). Understanding how and why the traits of different functional groups differ is also of practical use, given their unique effects on global biogeochemical cycles (Litchman et al. 2015). Relevant to our study, functional group identity has already been shown to explain variation in maximum growth rate of marine and freshwater phytoplankton, even after accounting for differences in cell volume (Litchman et al. 2007; Edwards et al. 2012). Consequently, testing the importance of functional group identity is a critical component of our efforts to reconcile metabolic theory and the Eppley curve.

In the current paper, we show that the temperaturedependence of growth rate is consistent with MTE, and weaker than suggested by earlier estimates of the Eppley curve, after correcting for the effects of cell size and functional group identity. This conclusion is based on a recently expanded data set (with $>4200$ observations), which includes observations used in earlier analyses (Eppley 1972; Bissinger et al. 2008) as well as additional values from the primary literature. It also required developing a way to compare these two competing frameworks, given their divergent 
statistical approaches. Our results suggest that the temperature-dependence utilized in many areas of aquatic ecology (including satellite observation algorithms and ecosystem models) need to be adjusted to provide accurate results. In particular, models of marine primary productivity that rely on the Eppley curve (e.g., Doney et al. 1996; Palmer and Totterdell 2001; Taucher and Oschlies 2011; Toseland et al. 2013; Stock et al. 2014), as previously parameterized (Eppley 1972; Bissinger et al. 2008) are likely to overestimate productivity increases driven by rising ocean temperatures.

\section{Methods}

\section{Data sources}

\section{Thermal tolerance curves}

We previously compiled data on estimates of population growth rates at varying temperatures from primary literature sources (Thomas et al. 2012, 2016). Subjected to rigorous quality control, these data consist of laboratory measurements that were made under reasonable light, nutrient, and salinity conditions (for details, see Thomas et al. 2012, 2016). In addition to those criteria, for the current analysis we also excluded any growth rates reported to be less than $0.1\left(\mathrm{~d}^{-1}\right)$, removing growth rate values near or below zero, which are very difficult to estimate reliably. We also excluded data from phytoplankton belonging to functional groups for which we had few measurements, leaving only diatoms, dinoflagellates, green algae, and cyanobacteria. Our data set includes marine, estuarine, and freshwater strains. Marine and freshwater species share many similarities in their thermal traits, although freshwater species have higher optimum temperatures (temperatures at which they achieve their maximum growth rate) and potentially wider thermal niches (Thomas et al. 2016). To focus on a biologically relevant range of temperatures, we also excluded a small number of growth rate estimates from temperatures $>40^{\circ} \mathrm{C}$. Finally, we excluded observations of taxa for which we were unable to find size estimates (next section). The remaining data serve as the foundation of subsequent analyses (4208 measurements in total; Supporting Information Table S.1) and are provided in the Supplementary Information.

\section{Cell size data}

Because papers supplying data on temperature-dependent growth rates rarely include corresponding estimates of cell size, we gathered estimates from a range of alternative sources, similar to the approach of Sal et al. (2015). The most common metric of size in phytoplankton is cell volume, typically estimated by calculating the mean volume of cells in a culture based on their three-dimensional shape and linear dimensions (Hillebrand et al. 1999). Previous papers have assembled cell volumes for freshwater and marine species (Litchman et al. 2009; Edwards et al. 2011). With these resources, and a new compilation of size data for $>1200$ freshwater species (Kremer et al. 2014), we obtained cell volume estimates for $\sim 92 \%$ of the taxa represented in our growth rate data set. Within functional groups, coverage of size estimates in our original growth data set ranged from a low of $81 \%$ (dinoflagellates) to a high of $98 \%$ (diatoms). Biovolume data and metadata are provided in the Supporting Information. Cell volume estimates (in $\mu \mathrm{m}^{3}$ ) were converted into estimates of dry weight (in $\mu \mathrm{g}$ ) using the relationship Mass $=0.47^{*}(\text { Volume })^{0.99} \times 10^{-6}$, after Reynolds (2006, p. $25)$. This is a standard relationship, although the exact connection between cell size and dry weight can vary significantly between cells and species, depending on the size of internal vacuoles and storage (Reynolds 2006), and other conversions have been proposed (e.g., Strathmann 1967; Menden-Deuer and Lessard 2000). In the analyses we present, we have focused on $\ln$ (mass) as our measure of phytoplankton body size, using the Reynolds relationship. However, analyses based on $\ln$ (cell volume) and estimates of mass following Strathmann (1967) yielded similar results. Finally, we also acknowledge that cell size varies significantly due to intra-specific variation and environmental effects. As a result, the literature-based size estimates we have obtained may differ from the true size of cells during the actual growth assays. This likely introduces additional uncertainty to our analyses, but is unavoidable.

\section{Eppley re-analysis \\ Prior analyses}

The Eppley curve attempts to capture an upper envelope bounding maximum growth rates as an exponential function of temperature. Bissinger et al. (2008) used regression techniques to rigorously estimate this envelope. Whereas standard linear regression models the relationship between covariate(s) and the mean of a response variable, quantile regression models variation in specific quantiles of the response variable. Models of extreme quantiles can be used to generate envelope functions (Cade and Noon 2003; Koenker 2005). Quantile regression is a useful approach, but it has some important limitations. In particular, acceptable fitting of extreme quantiles requires a great deal of data (Cade and Noon 2003; Koenker 2005). To avoid sample size issues, in our analyses we did not consider models with interactions between our covariates (size, temperature, functional group), for which we also lacked a priori hypotheses.

\section{Current analysis}

We used quantile regression to examine linear relationships between the $99^{\text {th }}$ quantile of $\ln (\mu)$ and covariates including temperature, $\ln (M)$, and functional group (Table 1). Linear models relating $\ln (\mu)$ and temperature are equivalent to assuming that growth rate, $\mu$, is exponentially related to temperature (Bissinger et al. 2008). We fit and evaluated all quantile regression models using the $[\mathrm{R}]$ package quantreg (version 5.19; Koenker 2015). To estimate the parameters of the Eppley curve, we used the entire growth rate data set 
described in 2.1 above. As with previous data used to address this question, our data set includes many examples where there are multiple growth rate observations per strain (ranging from 2 to 97), including sub-optimal growth rates estimated at temperatures above or below a strain's optimum temperature. Previous studies ignore this issue, but these measurements create a lack of independence among observations. Such situations are typically handled using a mixed effects model; however, there are no accessible methods combining mixed effects models and quantile regression. As a compromise, we used weighted quantile regression to ensure that each species contributes equally to the analysis independent of how many times they were measured. We calculated weights, $w_{i}$, for the observations associated with a particular strain $i$ as $w_{i}=1 /$ (\# of observations for strain $i$ ). In strains with many observations, each individual observation will consequently contributes less to the overall analysis. Ultimately, this enables us to avoid biasing quantile regressions towards intensively studied species or strains of phytoplankton. Code for these, and subsequent analyses, is provided in the Supporting Information. We also repeated our analyses after removing a few very high growth rate estimates, to check the sensitivity of the quantile regression fits to potential outliers (results not shown). The results did not change significantly, perhaps due to the higher level of uncertainty already inherent in regressions of extreme quantiles.

\section{Metabolic theory analysis Prior analyses}

Tests of metabolic theory differ in several significant ways from the Eppley-style analyses described in 2.2, including both the data and statistical methods they employ. Understanding and resolving these differences is essential to our work reconciling MTE with the Eppley curve, so we discuss the three major differences here. First, while Eppley analyses use all available growth rate data, MTE analyses ideally focus on just the highest observed growth rate per strain or species. While this dramatically reduces the amount of data used, MTE analyses do not depend on extreme quantile regressions, so they are inherently less sensitive to small sample sizes. Second, while the Eppley curve models maximum growth rate, $\mu_{\max }$ as a function of $\exp (T)$, MTE uses a Boltzmann term, which depends on $\exp (1 / T)$. More specifically, combining the temperature- and size- (or mass-) dependence of growth yields (after Savage et al. 2004a):

$$
\mu_{\max }=\mu_{0} M^{\alpha} e^{-E / k T}
$$

where $\mu_{0}$ is a normalization constant or intercept term, and other variables are as defined previously. We reconcile these differences using an approximation discussed later, in "Connecting Eppley and MTE analyses" section. Third, although the effects of mass and temperature can be combined in a single equation as in (Eq. 2), tests of MTE typically examine each effect separately. For example, a masscorrected growth rate will be regressed against temperature (assuming the theoretical value of $\alpha=-1 / 4$ is true), while a temperature-corrected growth rate will be regressed against mass (assuming $E=0.32 \mathrm{eV}$; or $0.65 \mathrm{eV}$ for heterotrophs). These bivariate analyses are necessary in MTE analyses that rely on "standardized major axis regression" or SMA (Warton et al. 2006; Edwards et al. 2015), a commonly used MTE technique that does not easily accommodate multiple covariates. While there are good reasons for conducting MTE tests this way (Warton et al. 2006), the approach has drawbacks: (1) estimating size and temperature effects separately, rather than conducting a multiple regression, can lead to parameter estimates with incorrect confidence intervals, and (2) SMA is incompatible with mixed effects models, yet data sets are often structured hierarchically, with repeated measurements of one or more species (Edwards et al. 2015).

\section{Current analysis}

To test metabolic theory's predictions, in light of these methodological issues and our over-arching goal of comparing MTE and the Eppley curve, we have adopted different approaches. First, we reduced our data so that it included only measurements of the highest observed growth rate for each strain, yielding $n=425$ values across 194 unique species (some species were represented by multiple strains). Second, rather than using SMA, we use linear mixed effects models. These have been used occasionally in MTE type analyses (e.g., O'Connor et al. 2007; Yvon-Durocher et al. 2012). With this approach, we can simultaneously investigate the effects of multiple covariates, while using a species-level random effect to account for the fact that $\sim 37 \%$ of the species in our data set are represented by multiple, separately measured strains. We examined models containing main effects of size, temperature, and functional group, as well as models where functional group interacted with size and/or temperature.

Specifically, models were fit in [R] using the function lmer in the lme4 package (version 1.1-10; Bates et al. 2014). Model comparison was used to select the best model based on AICc, which corrects for small sample sizes and converges on AIC for large sample sizes (Burnham and Anderson 2002). Estimates of $p$-values were obtained using a parametric bootstrapping approach, PBmodcomp, in the pbkrtest package (version 0.4-4; Halekoh and Højsgaard 2014). This method is resilient to violations of the asymptotic assumptions inherent in likelihood ratio and Wald tests (Halekoh and Højsgaard 2014), although all three methods yielded similar results. To assess the overall goodness of fit of models, we calculated estimates of conditional and marginal $\mathrm{R}^{2}$ using the function r.squaredGLMM in the R package MuMIn (version 1.13.4; Nakagawa and Schielzeth 2013). Finally, Tukey post-hoc comparisons were conducted using glht, also in the multcomp package (version 1.4-1; Hothorn et al. 2008), to 
Table 2. Results of the Eppley curve and MTE analyses. Additional details on model fits are presented as Supporting Information Tables S.2 and S.4. Variables match those defined in Table 1.

\begin{tabular}{|c|c|c|c|c|c|}
\hline \multicolumn{3}{|l|}{ Eppley curve results } & \multicolumn{3}{|c|}{ MTE results } \\
\hline Temperature & $b$ & 0.04738 & $1 /(\mathrm{kT})$ & $E$ & -0.300 \\
\hline Intercept (Cyanobacteria) & $a$ & -1.52173 & Intercept (Cyanobacteria) & $\mu_{0}$ & 10.50 \\
\hline Diatoms & $c_{3}$ & 0.69629 & Diatoms & $c_{3}$ & 0.992 \\
\hline
\end{tabular}

test for differences between functional groups as determined by our mixed effects model.

\section{Connecting Eppley and MTE analyses}

There are two remaining differences between the Eppley and MTE analyses that make it more difficult to compare their results. First, as we have mentioned above, these analyses use different temperature scales ( $T$ vs. $1 / T$ ), so their temperature coefficients ( $b$ and $E$ ) are not directly comparable. This issue can be resolved using a Taylor series approximation to express the MTE relationship as a function of temperature, $T$ (see Laidler 1984 and Supporting Information: Converting between MTE and Eppley). This leads to a simple conversion between the Eppley exponent $b$ and MTE's activation energy $E$ :

$$
b \approx \frac{E}{k T_{0}^{2}} \text { or } E \approx b \cdot k T_{0}^{2}
$$

where $T_{0}$ is 273. Mathematically, (Eq. 3) allows us to compare numerical estimates of the temperature-scaling of growth obtained from Eppley and MTE analyses. For example, we can take estimates of $b$, its standard error, and its 95\% confidence interval (all obtained by quantile regression), and convert them into activation energies. Similarly, an estimated activation energy coefficient from an MTE regression can be converted into an Eppley exponent. To test whether these values differ significantly from their theorized or previously estimated values (Table 1), we can use a simple two-tailed test of differences in slope (Zar 1999). Second, we note that the conversion in Eq. 3 simply allows us to quantitatively compare Eppley and MTE coefficients. Differences in these coefficients may be affected by the different statistical methods (quantile regression vs. linear regression) and data (all growth rates vs. maximum growth rates of individual strains) employed by each approach (Eppley and MTE, respectively). This is important to recall when interpreting results presented in the following sections.

\section{Results}

\section{Eppley results}

We found significant effects of temperature, cell mass, and functional group identity on the maximum growth rates of phytoplankton (Table 2, Supporting Information Table S.2), using methods from "Current analysis" section. The model including these three main effects performed better than a set of simpler models (Supporting Information Table S.3), based on AICc comparison. As expected, growth rates increased with temperature (Fig. $1 \mathrm{~A} ; b=0.047, p<0.0001$, $95 \% \mathrm{CI}=\{0.034,0.060\}$ ), but decreased with cell size (Fig. $1 \mathrm{~B}$; slope $=-0.084, p<0.0001,95 \% \mathrm{CI}=\{-0.125,-0.043\})$. Functional groups displayed significantly different baseline capacities for growth: across temperatures and cell masses, the cyanobacteria and dinoflagellates exhibited much lower maximum growth rates than diatoms and green algae (Fig. 1; Supporting Information Table S.2). In fact, cyanobacteria and dinoflagellates never display maximum growth rates as high as in previous estimates of the Eppley curve (Fig. 2), while diatoms and green algae do so only at low temperatures. These differences among groups cannot be attributed to differences in size between groups (Supporting Information Fig. S.1), as cell mass was included in the same model. We were unable to test for an interaction between temperature and functional group (i.e., distinct Eppley exponents for each group), due to the data-hungry nature of extreme quantile regressions. However, this question is addressed in the MTE analyses below.

\section{MTE results}

Performing an MTE analysis (see "Prior analyses" section above), we again found significant main effects of temperature, cell mass, and functional group identity on maximum growth rates (Fig. 3; Table 2, Supporting Information Table S.4). In particular, temperature increased growth rates, with an activation energy of $E=0.30 \mathrm{eV} \quad(p<0.001, \quad 95 \%$ $\mathrm{CI}=0.233$ to 0.368; Supporting Information Table S.4), which is quite similar to the theoretically predicted value of $0.32 \mathrm{eV}$ (Table 1). While mass had a negative effect on growth rate $(\alpha=-0.054 ; p<0.005,95 \% \mathrm{CI}=-0.089$ to $-0.018)$, the strength of this effect was much weaker than predicted $(\alpha=-0.25$; Savage et al. 2004a,b). As with the Eppley analysis, functional groups differed from each other in their capacity for growth (i.e., growth rate at $0^{\circ} \mathrm{C} ; p<0.001$ ), except for cyanobacteria and dinoflagellates (Supporting 


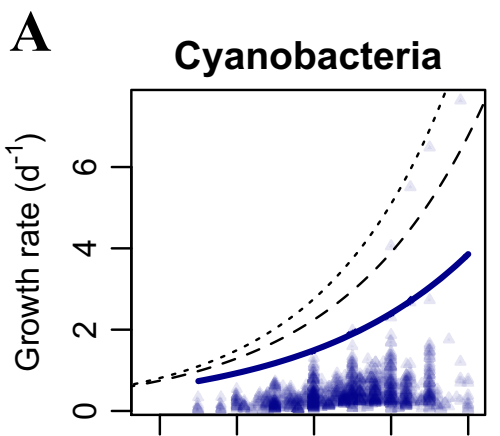

Dinoflagellates

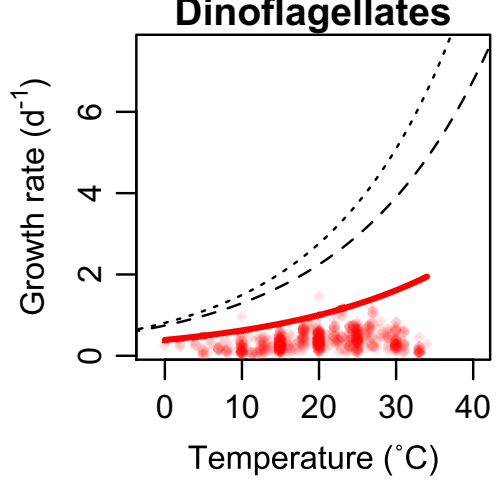

Diatoms

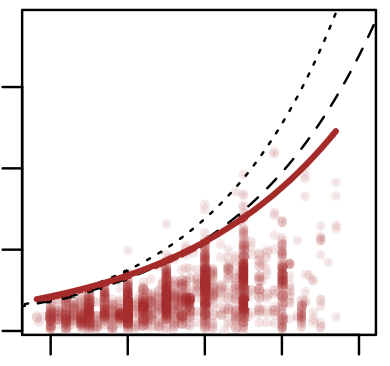

Greens

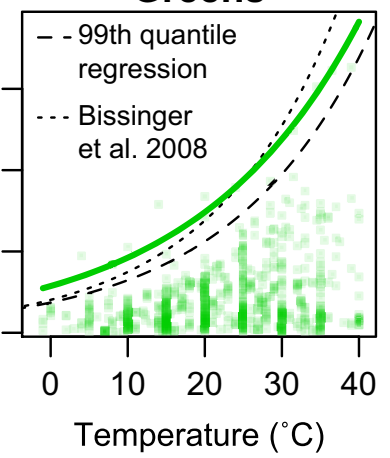

B

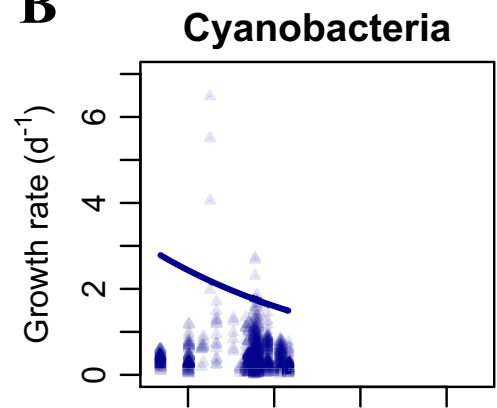

Dinoflagellates

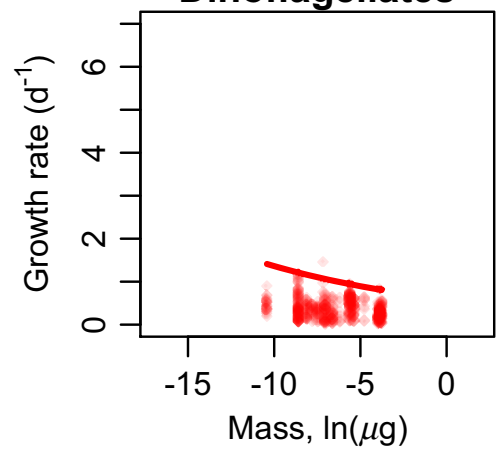

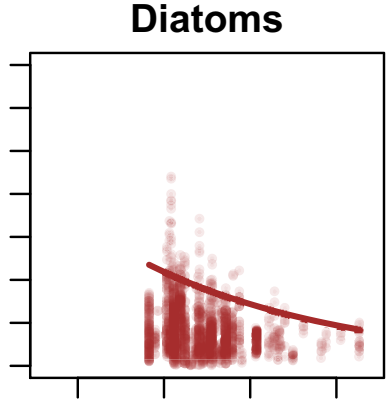

Greens

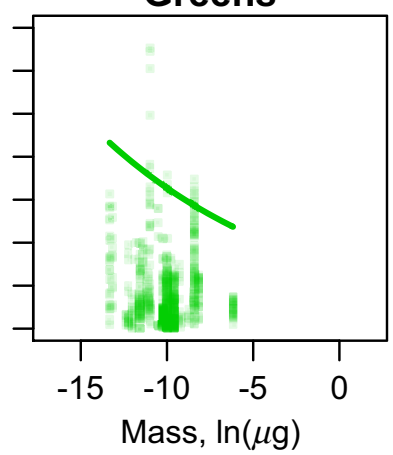

Fig. 1. (A) The Eppley curves of phytoplankton functional groups vary substantially (solid lines). Plotted regressions are from a weighted, $99^{\text {th }}$ quantile regression including main effects of temperature, functional group, and mass. For visualization purposes, each curve was drawn using the mean mass of the corresponding functional group. Individual observations contributing to the analysis are indicated by partially transparent points. Notably, temperature-scaling in our new estimate of the Eppley curve is weaker than previously estimated by Bissinger et al. (2008) (dotted line). For reference, we also plot the results of applying Bissinger et al.'s approach, ignoring size and functional group, to our data set (dashed line). (B) Growth rates simultaneously decline with increasing cell mass; these plots show the size effect produced by the same $99^{\text {th }}$ quantile regression as displayed in panel A. For visualization, each curve was drawn using the mean temperature of the corresponding functional group within our data set.

Information Table S.5). We also fit two additional models testing for an interaction effect between functional group identity and temperature, or size and temperature, respectively. However, AICc model comparison showed that neither of these more complex models were improvements (Supporting Information Table S.6). This implies that phytoplankton share a common activation energy across functional groups and cell sizes. This is consistent with the MTE literature, which has repeatedly identified similar scaling exponents across major branches of life, and does not predict interactive effects between size and temperature (e.g., Brown et al. 2004; Savage et al. 2014a).

\section{Eppley vs. MTE comparison}

Finally, we turn to comparing our newly estimated effects of temperature on maximum growth rate (after accounting for both mass and functional group effects) with the values provided by MTE and previous estimates of the Eppley curve. We find that the temperature-dependence of population growth rate is significantly weaker than implied by previous estimates of the Eppley curve, but not statistically different than MTE predicts (Fig. 4; Supporting Information Table S.7). This result applies whether we use the parameters estimated from the quantile regression approach, or MTE methods (Supporting Information Table S.7). Interestingly, when we fit a simple, un-weighted quantile regression of maximum growth rate against temperature alone (reproducing Bissinger et al. 2008's re-analysis of the Eppley curve), we obtain a higher estimate of $b$ (0.055; $p<0.00001,95 \%$ $\mathrm{CI}=\{0.049,0.061\}$; Supporting Information Table S.8). This corresponds to a temperature-scaling effect that is weaker than prior estimates of the Eppley curve, stronger than MTE predictions, and significantly distinct from both (Supporting Information Table S.7).

\section{Discussion and conclusion}

Characterizing the effects of temperature on organisms is central to understanding fundamental patterns in aquatic communities and ecosystems. This task is impeded by the existence of two popular frameworks (the Eppley curve and the metabolic theory of ecology) that describe the temperature-dependence of growth rate differently. Figuring out how to directly compare these paradigms is essential for advancing the study of phytoplankton communities and for refining contemporary models of primary productivity and 
global ecosystems. We have demonstrated how these two frameworks, with divergent histories and separate literatures, can be related ("Connecting Eppley and MTE analyses" section and "Eppley vs. MTE comparison section" above). From this foundation, our analyses show that the increase of population growth rate with temperature is weaker than previous characterizations of the Eppley curve, yet consistent with MTE predictions (Supporting Information Table S.7),

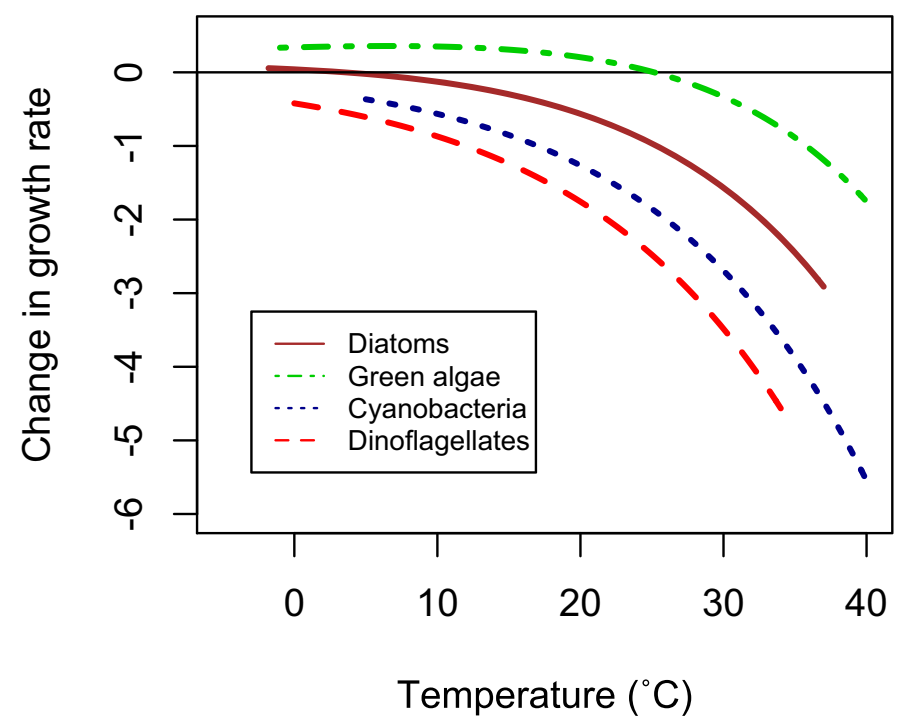

Fig. 2. Difference in predicted maximum population growth rate $\left(d^{-1}\right)$ relative to the Eppley curve as estimated by Bissinger et al. (2008). Although our results indicate that growth rate increases more slowly with temperature than in previous versions of the Eppley curve, functional groups have different intercepts. Consequently, at low temperatures, fast growing green algae and diatoms grow faster than previously predicted. In contrast, at high temperatures, all groups show substantially lower growth rates. [Color figure can be viewed at wileyonlinelibrary.com]

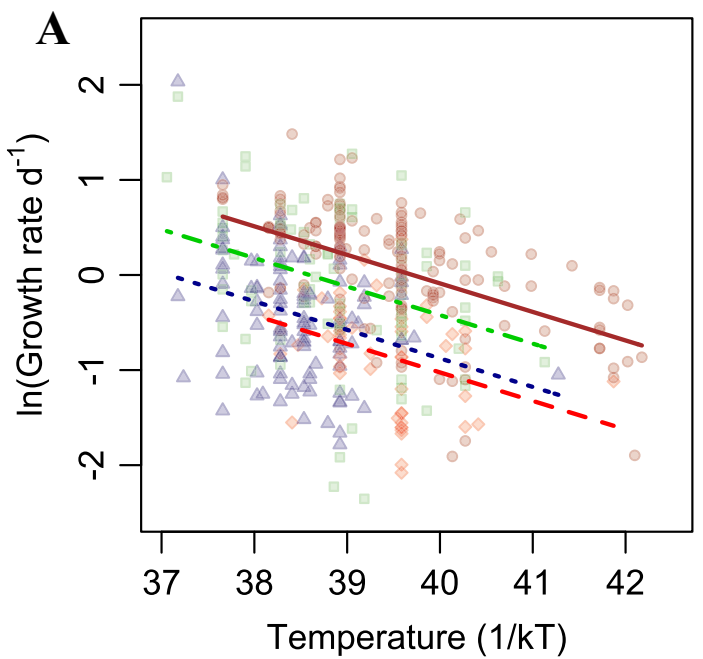

suggesting that maximum growth rate is indeed limited by the temperature sensitivity of photosynthesis. For a $10^{\circ} \mathrm{C}$ increase in temperature, previous estimates of the Eppley curve suggested that growth rates would increase by a factor of 1.88; in contrast, our new results suggest that this increase is smaller, around a factor of 1.53 (given Supporting Information Table S.3). We also found evidence that differences in size explain less variation in growth rate than MTE predictions suggest. Under the assumed MTE value of -0.25 for the size-scaling relationship, a decrease in cell mass of $\sim 3$ orders in magnitude would increase growth rates by a factor of $\sim 3.5$; in contrast, if the weaker relationship we obtained is true, growth rates would only increase by a factor of $\sim 1.5$ (given Supporting Information Table S.3).

Critically, our results also show that the slope of the temperature-scaling relationship is consistent across functional groups (Supporting Information Table S.6), although groups differ in their intercepts (maximum growth rate at $\mathrm{T}=0^{\circ} \mathrm{C}$ ). The invariant nature of this relationship across groups supports a central tenet of metabolic theory-that the temperature dependence of growth rate under replete conditions emerges from the shared biochemistry and thermodynamic constraints of all photoautotrophs. It also implies that increases in ocean temperatures will not change the growth rate hierarchy of functional groups, as their growth rate vs. temperature relationships will never cross. Despite the our new estimate of a weaker effect of temperature on growth rate, we found that diatoms and green algae have intercepts that are higher than the previously estimated intercept of the Eppley curve (Table 1 vs. Supporting Information Table S.2). This implies that models using older parameterizations of the Eppley curve to describe the growth of these functional groups will underestimate their growth rates at low temperatures, while over estimating their growth

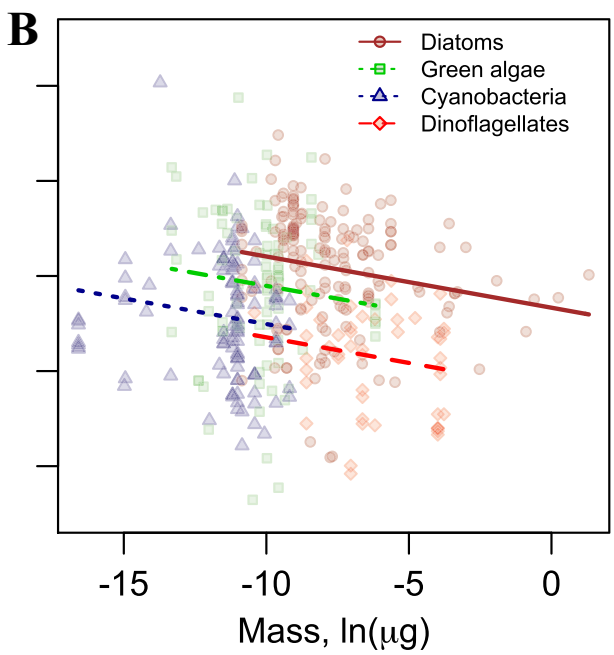

Fig. 3. Partial regression plots from the metabolic theory analysis showing the effects of: (A) temperature and (B) cell mass on growth rate, separated by a significant effect of functional group. 

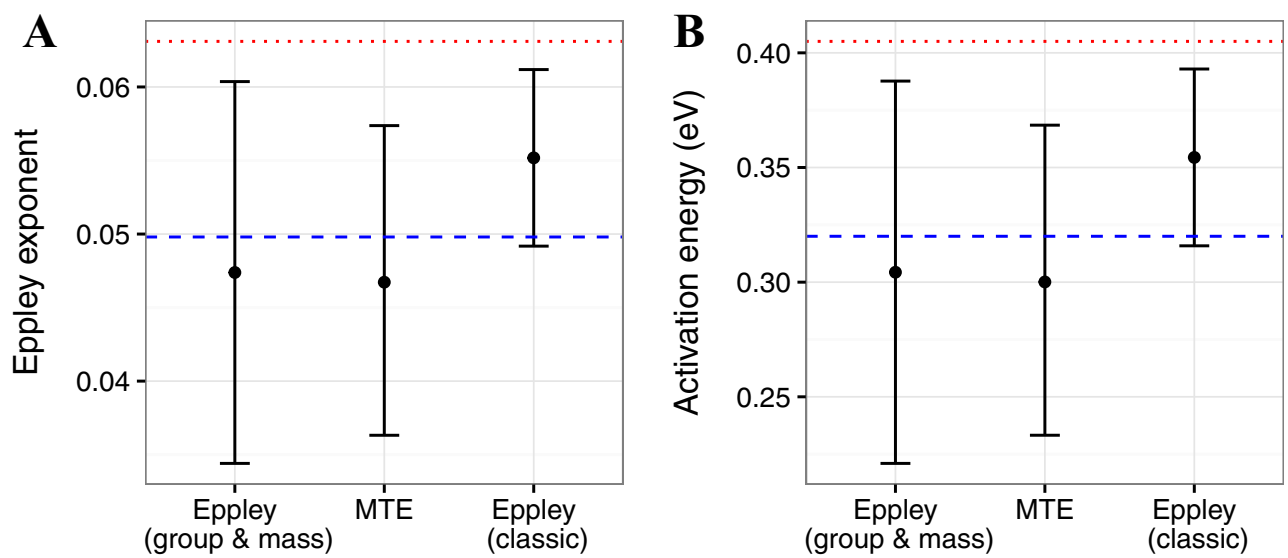

Fig. 4. We can illustrate the temperature-scaling coefficients obtained through Eppley or MTE analyses as either (A) an Eppley exponent or (B) an activation energy, due to the conversion derived in the Supporting Information. This allows us to compare our newly estimate coefficients (black dots, with 95\% confidence interval error bars) with predictions based on previous Eppley analyses (red dotted line; Bissinger et al. 2008) and theoretical MTE predictions (blue dashed line). Our Eppley analysis (accounting for functional group and mass) and the MTE analysis yield coefficients that are significantly lower than previous Eppley estimates, but not significantly different from the MTE prediction (Supporting Information Table S.7). However, an Eppley analysis ignoring functional group and mass ("Epply classic") yields a larger temperature-scaling coefficient that differs significantly from the MTE and Eppley predictions (Supporting Information Table S.7). [Color figure can be viewed at wileyonlinelibrary.com]

rates at high temperatures (Fig. 2). Collectively, our results highlight the importance of developing a conceptually and quantitatively accurate framework describing interactions between cellular physiology, environmental factors (like temperature), and key ecological parameters (such as species' population growth rate).

Our efforts to resolve the differences between the Eppley and MTE frameworks depended critically on accounting for the effects of cell size and functional group. Although the possibility of these effects was discussed in previous work, it was not directly addressed (Eppley 1972; Bissinger et al. 2008). When we performed a $99^{\text {th }}$ quantile regression relating population growth rate to temperature, but ignoring both of these factors, we obtained an estimate of the temperature effect that was significantly larger than the MTE prediction ("Eppley vs. MTE comparison" section; Supporting Information Table S.7). We believe that this discrepancy likely arises from what is essentially a model specification error. Basically, there is heterogeneity in the observed growth rates that a temperature-only model cannot capture, as it is attributable to functional group identity and cell size. Otherwise unexplained, this heterogeneity ends up biasing estimates of the temperature-scaling coefficient in quantile regressions, especially at extreme quantiles (e.g., the $99^{\text {th }}$ quantile), yielding higher estimates of the temperature-scaling coefficient. We present additional results and discussion of this statistical issue (see Supporting Information: Evaluation of quantile regression stability). This effect may explain why previous analyses of the Eppley curve (e.g., Bissinger et al. 2008) found temperature coefficients that are larger than predicted by MTE analyses.

Functional group identity and cell size are also interesting in their own right, not just because they are important for obtaining rigorous estimates of temperature effects. We turn now to discussing cell size and mass. Although Eppley noted that cell size could have significant effects on growth rate (1972), his analysis and the resulting Eppley curve do not account for size. In contrast, metabolic theory makes explicit, quantitative predictions for the effect of mass on individual metabolism and, subsequently, population growth rate. While we found that growth rate decreases with cell size, consistent with general knowledge, the rate of decline was much weaker than MTE predicts $(-0.054$ rather than -0.25$)$. This result is consistent with previous publications on phytoplankton (review of Chisholm 1992; more recently, Marañón 2008; Chen and Liu 2011; Sal et al. 2015). Note that in these and other studies, it is important to distinguish between examples of the temperature sensitivity of production, respiration, and growth rate.

There are several reasons why we might observe size scaling relationships in phytoplankton that deviate from MTE predictions: (1) Cell size varies significantly within and among strains and species, often in response to environmental conditions such as temperature (e.g., Finkel et al. 2009). We used mean cell size estimates taken from the literature, rather than values taken during growth rate assays, as do other studies (e.g., Edwards et al. 2011, 2012; Sal et al. 2015). This introduces noise to our analysis, which would reduce the strength of observed relationships. (2) The strength of size-scaling relationships can be sensitive to the metric of size used. For example, based on in situ data, López-Urrutia et al. (2006) found that rates of both net production and respiration scaled nearly isometrically (i.e., with slope $=1$ ) with the total mass of carbon in cells, deviating from MTE predictions. However, using cell biovolume instead of mass yielded a relationship more consistent with the $3 / 4$ prediction, which 
they attributed to the allometric relationship between the volume and carbon content of cells. These conversions are problematic: multiple relationships exist for converting between cell volume and mass in carbon (e.g., Strathmann 1967; Menden-Deuer and Lessard 2000; Reynolds 2006), and the choice of conversion can affect estimates of scaling relationships (Marañón 2008). However, in our analysis, we found very little difference in the size-scaling coefficient whether we used $\ln$ (biovolume) or $\ln$ (mass), calculating mass using two different conversions (Supporting Information Table S.9; Strathman 1967; Reynolds 2006). In the end, due to variation in the physiology of different species and functional groups (e.g., vacuoles affect cell size, but hold little carbon), a universally applicable conversion based on allometry may not exist; (3) Finally, there are important aspects of the biology of phytoplankton not incorporated into MTE's predictions that could lead to a weaker size-scaling relationship (Chisholm 1992; Marañón 2015). Larger cells are generally assumed to have lower surface area to volume ratios and to experience increased self-shading. As a result, growth rate is thought to decrease with size, as larger cells struggle to use light efficiently and to obtain sufficient nutrients and distribute them internally through diffusion. However, changes in cell shape with increasing size can alleviate these constraints and weaken the size-scaling relationship (Marañón 2008). Additionally, small cells may experience limited growth rates because unavoidable investments in non-scalable nitrogenous compounds such as DNA result in reduced biosynthetic capacity. Depending on their strength and shape, the combined effects of opposing tradeoffs between being small and large could produce a size-scaling relationship that is weaker than the MTE proposed $-1 / 4$, or even result in a nonlinear relationship (Marañón et al. 2013; Marañón 2015). Additional research is clearly needed to determine the strength and shape of size-scaling relationships, efforts that will benefit from controlling for confounding effects of environment and phylogenetic relationships among taxa (e.g., Sal et al. 2015).

We turn next to considering functional group effects: both the Eppley and MTE style analyses indicated that functional group identity explained significant variation in the intercept (but not the slopes) of the temperature- and sizescaling relationships (Figs. 1, 3; Supporting Information Tables S.3, S.6). Variation in intercepts among taxonomic groups has been frequently documented in MTE studies (e.g., Brown et al. 2004) and occasionally discussed (Gillooly et al. 2001). However, to our knowledge, no rigorous mechanisms explaining these differences have been proposed, creating opportunities for the future development of metabolic theory. The differences in growth rates we observe among phytoplankton groups are consistent with previous work (Edwards 2012), adding to a growing literature on the distinct traits of functional groups (Litchman and Klausmeier 2008; Finkel et al. 2009; Edwards et al. 2012, 2015; Thomas et al. 2016). The slow growth rate of dinoflagellates has been noted previously (Tang 1995), especially relative to diatoms (Chisholm 1992). The source of these differences may be linked to fundamental physiological distinctions. For example, genome size varies across taxonomic groups: dinoflagellates in particular have enormous genomes, and consistently lower growth rates (e.g., Litchman et al. 2007; Oliver et al. 2007). Functional groups also differ in their ecological strategies, in ways that may influence their growth rate. Dinoflagellates are often mixotrophic, but our data reflect their ability to grow autotrophically. The ability to both photosynthesize and consume other organisms may come at the expense of rapid growth. In contrast, diatoms can grow very rapidly, perhaps consistent with selection to take advantage of pulses of nutrients supplied by seasonal turnover or other events. In general, because the functional group differences we document are consistent across dozens of species and a wide range of temperatures, the underlying mechanisms seem likely to be tied to evolutionarily conserved features (Thomas et al. 2016).

Together, developing an accurate knowledge of the temperature-dependence of phytoplankton growth, as well as the distinct properties of functional groups, is important for developing rigorous ecosystem models. These models often attempt to describe the dynamics of entire ecosystems across broad spatial and temporal ranges by reducing the complexity of phytoplankton and zooplankton communities into a handful of representative groups, usually based on size and/or functional group identity. For these models to accurately forecast ecosystem dynamics both now and in a future inevitably influenced by climate change, it is essential that they use a quantitatively accurate temperature-scaling. Balancing tradeoffs between complexity and computational cost, they must also employ functional groups that capture the most important differences among species. Consequently, it is important to know which relationships apply broadly (ie, growth rates increase with temperature according to $\sim 0.32 \mathrm{eV}$ ) and which differ by group. For example, our results suggest that, at the same temperature, communities dominated by different functional groups will have substantially different maximum growth rates, and hence, productivities (Fig. 1; growth rate of green algae $>$ diatoms $>$ cyanobacteria $>$ dinoflagellates). While growth rates will increase across temperatures, this hierarchy will not change. Collectively, these results present an opportunity for further refining the structure and parameterization of ecosystem models.

This paper is an attempt to resolve the conflict between distinct but related bodies of research that describe how phytoplankton growth rates scale with temperature, by uniting divergent methodologies and exploring an extensive empirical data set. We have shown that metabolic theory provides a more quantitatively accurate, and conceptually rigorous, description of the temperature-dependence of growth, subject to additional effects of cell size and functional group identity. Understanding the physiological and mechanistic basis for the differences among functional groups, and 
dissecting these patterns using more nuanced phylogenetic methods, represent important goals. Other important avenues for further research include challenging the mechanistic basis of metabolic theory (e.g., Clarke 2006), and attempting to relate the inter-specific scaling patterns explored in the present paper to the effects of temperature on the growth of individual species. Ultimately, it is our hope that the current results will strengthen the theoretical foundation and parameterization of research, including ecosystem modeling, that depends on understanding how changing environmental conditions affect phytoplankton community composition and ecosystem function.

\section{References}

Allen, A. P., J. F. Gillooly, and J. H. Brown. 2005. Linking the global carbon cycle to individual metabolism. Funct. Ecol. 19: 202-213. doi:10.1111/j.1365-2435.2005.00952.x

Angilletta, M. J., R. B. Huey, Jr., and M. R. Frazier. 2010. Thermodynamic effects on organismal performance: Is hotter better? Physiol. Biochem. Zool. 83: 197-206. doi: 10.1086/648567

Antoine, D., J. M. André, and A. Morel. 1996. Oceanic primary production 2. Estimation at global scale from satellite (coastal zone color scanner) chlorophyll. Global Biogeochem. Cycles 10: 57-69. doi:10.1029/95GB02832

Banavar, J. R., A. Maritan, and A. Rinaldo. 1999. Size and form in efficient transportation networks. Nature 399: 130-132. doi:10.1038/20144

Bates, D., M. Machler, B. Bolker, and S. Walker. 2014. Fitting linear mixed-effects models using lme4. ArXiv E-Prints. Available from http://arxiv.org/abs/1406.5823

Bec, B., Y. Collos, A. Vaquer, D. Mouillot, and P. Souchu. 2008. Growth rate peaks at intermediate cell size in marine photosynthetic picoeukaryotes. Limnol. Oceanogr. 53: 863-867. doi:10.4319/1o.2008.53.2.0863

Behrenfeld, M. J., and P. G. Falkowski. 1997. Photosynthetic rates derived from satellite-based chlorophyll concentration. Limnol. Oceanogr. 42: 1-20. doi:10.4319/lo.1997.42.1.0001

Bickel, P. J., E. A. Hammel, and J. W. O'Connell. 1975. Sex bias in graduate admissions: Data from Berkeley. Science 187: 398-404. doi:10.1126/science.187.4175.398

Bissinger, J. E., D. J. S. Montagnes, J. Sharples, and D. Atkinson. 2008. Predicting marine phytoplankton maximum growth rates from temperature: Improving on the Eppley curve using quantile regression. Limnol. Oceanogr. 53: 487-493. doi:10.4319/lo.2008.53.2.0487

Brown, J. H., J. F. Gillooly, A. P. Allen, V. M. Savage, and G. B. West. 2004. Toward a metabolic theory of ecology. Ecology 85: 1771-1789. doi:10.1890/03-9000

Brush, M. J., J. W. Brawley, S. W. Nixon, and J. N. Kremer. 2002. Modeling phytoplankton production: Problems with the Eppley curve and an empirical alternative. Mar. Ecol. Prog. Ser. 238: 31-45. doi:10.3354/meps238031
Burnham, K. P., and D. R. Anderson. 2002. Model selection and multimodel inference: A practical informationtheoretic approach, 2nd ed. Springer-Verlag.

Cade, B. S., and B. R. Noon. 2003. A gentle introduction to quantile regression for ecologists. Front. Ecol. Environ. 1: 412-420. doi:10.1890/1540-9295(2003)001[0412:AGITQR]2.0.CO;2

Chen, B. Z., and H. B. Liu. 2010. Relationships between phytoplankton growth and cell size in surface oceans: Interactive effects of temperature, nutrients, and grazing. Limnol. Oceanogr. 55: 965-972. doi:10.4319/lo.2010.55.3.0965

Chen, B. Z., and H. B. Liu. 2011. Comment: Unimodal relationship between phytoplankton-mass-specific growth rate and size: A reply to the comment by Sal and LópezUrrutia (2011). Limnol. Oceanogr. 56: 1956-1958. doi: 10.4319/lo.2011.56.5.1956

Chisholm, S. W. 1992. Phytoplankton size, p. 213-237. In P. G. Falkowski and A. D. Woodhead [eds.], Primary productivity and biogeochemical cycles in the sea. Plenum Press.

Clark, J. S., and others. 2011. Individual-scale variation, species-scale differences: Inference needed to understand diversity. Ecol. Lett. 14: 1273-1287. doi:10.1111/j.14610248.2011.01685.x

Clarke, A. 2006. Temperature and the metabolic theory of ecology. Funct. Ecol. 20: 405-412. doi:10.1111/j.13652435.2006.01109.x

Doney, S. C., D. M. Glover, and R. G. Najjar. 1996. A new coupled, one-dimensional biological-physical model for the upper ocean: Applications to the JGOFS Bermuda Atlantic Time-Series Study (BATS) site. Deep-Sea Res. II 43: 591-624. doi:10.1016/0967-0645(95)00104-2

Edwards, K. F., C. A. Klausmeier, and E. Litchman. 2011. Evidence for a three-way trade-off between nitrogen and phosphorus competitive abilities and cell size in phytoplankton. Ecology 92: 2085-2095. doi:10.1890/110395.1

Edwards, K. F., M. K. Thomas, C. A. Klausmeier, and E. Litchman. 2012. Allometric scaling and taxonomic variation in nutrient utilization traits and maximum growth rate of phytoplankton. Limnol. Oceanogr. 57: 554-566. doi:10.4319/1o.2012.57.2.0554

Edwards, K. F., M. K. Thomas, C. A. Klausmeier, and E. Litchman. 2015. Light and growth in marine phytoplankton: Allometric, taxonomic, and environmental variation. Limnol. Oceanogr. 60: 540-552. doi:10.1002/lno.10033

Eppley, R. W. 1972. Temperature and phytoplankton growth in the sea. Fish. Bull. 70: 41063-41085.

Finkel, Z. V., J. Beardall, K. J. Flynn, A. Quigg, T. A. V. Rees, and J. A. Raven. 2009. Phytoplankton in a changing world: Cell size and elemental stoichiometry. J. Plankton Res. 32: 119-137. doi:10.1093/plankt/fbp098

Gillooly, J. F., J. H. Brown, G. B. West, V. M. Savage, and E. L. Charnov. 2001. Effects of size and temperature on metabolic rate. Science 293: 2248-2251. doi:10.1126/ science. 1061967 
Gillooly, J. F., E. L. Charnov, G. B. West, V. M. Savage, and J. H. Brown. 2002. Effects of size and temperature on developmental time. Nature 417: 70-73. doi:10.1038/417070a

Halekoh, U., and S. Højsgaard. 2014. A Kenward-Roger approximation and parametric bootstrap methods for tests in linear mixed models-the $\mathrm{R}$ package pbkrtest. J. Stat. Softw. 59: 1-32. doi:10.18637/jss.v059.i09

Hillebrand, H., C. D. Dürselen, D. Kirschtel, U. Pollingher, and T. Zohary. 1999. Biovolume calculation for pelagic and benthic microalgae. J. Phycol. 35: 403-424. doi: 10.1046/j.1529-8817.1999.3520403.x

Hothorn, T., F. Bretz, and P. Westfall. 2008. Simultaneous inference in general parametric models. Biom. J. 50: 346363. doi:10.1002/bimj.200810425

Koenker, R. 2005. Quantile regression. Cambridge Univ. Press.

Koenker, R. 2015. quantreg: Quantile Regression. R package version 5.19. Available from https://CRAN.R-project.org/ package $=$ quantreg

Kremer, C. T., J. P. Gillette, L. G. Rudstam, and P. Brettum. 2014. A compendium of cell and natural unit biovolumes for $>1200$ freshwater phytoplankton species. Ecology 95: 2984. doi:10.1890/14-0603.1

Laidler, K. J. 1984. The development of the Arrhenius equation. J. Chem. Educ. 61: 494-498. doi:10.1021/ed061p494

Litchman, E., C. A. Klausmeier, O. M. Schofield, and P. G. Falkowski. 2007. The role of functional traits and tradeoffs in structuring phytoplankton communities: Scaling from cellular to ecosystem level. Ecol. Lett. 10: 11701181. doi:10.1111/j.1461-0248.2007.01117.x

Litchman, E., and C. A. Klausmeier. 2008. Trait-based community ecology of phytoplankton. Ann. Rev. of Ecol., Evolution, and Systematics 39: 615-639. doi:10.1146/ annurev.ecolsys.39.110707.173549

Litchman, E., C. A. Klausmeier, and K. Yoshiyama. 2009. Contrasting size evolution in marine and freshwater diatoms. Proc. Natl. Acad. Sci. USA 106: 2665-2670. doi: 10.1073/pnas.0810891106

Litchman, E., P. de Tezanos Pinto, K. F. Edwards, C. A. Klausmeier, C. T. Kremer, and M. K. Thomas. 2015. Global biogeochemical impacts of phytoplankton: A trait-based perspective. J. Ecol. 103: 1384-1396. doi:10.1111/1365-2745.12438

López-Urrutia, Á., E. San Martin, R. P. Harris, and X. Irigoien. 2006. Scaling the metabolic balance of the oceans. Proc. Natl. Acad. Sci. USA 103: 8739-8744. doi: 10.1073/pnas.0601137103

Marañón, E. 2008. Inter-specific scaling of phytoplankton production and cell size in the field. J. Plankton Res. 30: 157-163. doi:10.1093/plankt/fbm087

Marañón, E. 2015. Cell size as a key determinant of phytoplankton metabolism and community structure. Ann. Rev. Mar. Sci. 7: 241-264. doi:10.1146/annurev-marine010814-015955

Marañón, E., P. Cermeño, D. C. López-Sandoval, T. Rodríguez-Ramos, C. Sobrino, M. Huete-Ortega, J. M.
Blanco, and J. Rodríguez. 2013. Unimodal size scaling of phytoplankton growth and the size dependence of nutrient uptake and use. Ecol. Lett. 16: 371-379. doi:10.1111/ ele. 12052

Menden-Deuer, S., and E. J. Lessard. 2000. Carbon to volume relationships for dinoflagellates, diatoms, and other protist plankton. Limnol. Oceanogr. 45: 569-579. doi: 10.4319/lo.2000.45.3.0569

Morel, A. 1991. Light and marine photosynthesis: A spectral model with geochemical and climatological implications. Prog. Oceanogr. 26: 263-306. doi:10.1016/00796611(91)90004-6

Nakagawa, S., and H. Schielzeth. 2013. A general and simple method for obtaining $\mathrm{R}^{2}$ from Generalized Linear Mixedeffects Models. Methods Ecol. Evol. 4: 133-142. doi: 10.1111/j.2041-210x.2012.00261.x

O'Connor, M. I., J. F. Bruno, and S. D. Gaines. 2007. Temperature control of larval dispersal and the implications for marine ecology, evolution, and conservation. Proc. Natl. Acad. Sci. USA 104: 1266-1271. doi:10.1073/pnas.0603422104

Oliver, M. J., D. Petrov, D. Ackerly, P. Falkowski, and O. M. Schofield. 2007. The mode and tempo of genome size evolution in Eukaryotes. Genome Res. 17: 594-601. doi: 10.1101/gr.6096207

Palmer, J. R., and I. J. Totterdell. 2001. Production and export in a global ocean ecosystem model. Deep-Sea Res. Part I 48: 1169-1198. doi:10.1016/S0967-0637(00)00080-7

Raven, J. A. 1994. Why are there no picoplanktonic $\mathrm{O}_{2}$ evolvers with volumes less than $10^{-19} \mathrm{~m}^{3}$ ? J. Plankton Res. 16: 565-580. doi:10.1093/plankt/16.5.565

Reynolds, C. S. 2006. Ecology of phytoplankton. Cambridge Univ. Press.

Rose, J. M., and D. A. Caron. 2007. Does low temperature constrain the growth rates of heterotrophic Protists? Evidence and implications for algal blooms in cold waters. Limnol. Oceanogr. 52: 886-895. doi:10.4319/lo.2007.52.2.0886

Sal, S., and Á. López-Urrutia. 2011. Comment: Temperature, nutrients, and the size-scaling of phytoplankton growth in the sea. Limnol. Oceanogr. 56: 1952-1955. doi: 10.4319/1o.2011.56.5.1952

Sal, S., L. Alonso-Sáez, J. Bueno, F. C. García, and Á. LópezUrrutia. 2015. Thermal adaptation, phylogeny, and the unimodal size scaling of marine phytoplankton growth. Limnol. Oceanogr. 60: 1212-1221. doi:10.1002/lno.10094

Savage, V. M., J. F. Gillooly, J. H. Brown, G. B. West, and E. L. Charnov. 2004a. Effects of body size and temperature on population growth. Am. Nat. 163: 429-441. doi: $10.1086 / 381872$

Savage, V. M., J. F. Gillooly, W. H. Woodruff, G. B. West, A. P. Allen, B. J. Enquist, and J. H. Brown. 2004b. The predominance of quarter-power scaling in biology. Funct. Ecol. 18: 257-282. doi:10.1111/j.0269-8463.2004.00856.x

Stock, C. A., J. P. Dunne, and J. G. John. 2014. Global-scale carbon and energy flows through the marine planktonic food 
web: An analysis with a coupled physical-biological model. Prog. Oceanogr. 120: 1-28. doi:10.1016/j.pocean.2013.07.001

Strathmann, R. 1967. Estimating the organic carbon content of phytoplankton from cell volume or plasma volume. Limnol. Oceanogr. 12: 411-418. doi:10.4319/lo.1967.12.3.0411

Tang, E. P. Y. 1995. The allometry of algal growth rates. J. Plankton Res. 17: 1325-1335. doi:10.1093/plankt/17.6.1325

Taucher, J., and A. Oschlies. 2011. Can we predict the direction of marine primary production change under global warming? Geophys. Res. Lett. 38: 1-6. doi:10.1029/ 2010GL045934

Tett, P., S. I. Heaney, and M. R. Droop. 1985. The Redfield ratio and phytoplankton growth rate. J. Mar. Biol. Assoc. UK 65: 487-504. doi:10.1017/S0025315400050566

Thomas, M. K., C. T. Kremer, C. A. Klausmeier, and E. Litchman. 2012. A global pattern of thermal adaptation in marine phytoplankton. Science 338: 1085-1088. doi: 10.1126/science. 1224836

Thomas, M. K., C. T. Kremer, and E. Litchman. 2016. Environment and evolutionary history determine the global biogeography of phytoplankton temperature traits. Glob. Ecol. Biogeogr. 25: 75-86. doi:10.1111/geb.12387

Toseland, A., and others. 2013. The impact of temperature on marine phytoplankton resource allocation and metabolism. Nat. Clim. Chang. 3: 979-984. doi:10.1038/ NCLIMATE1989

Warton, D. I., I. J. Wright, D. S. Falster, and M. Westoby. 2006. Bivariate line-fitting methods for allometry. Biol. Rev. 81: 259-291. doi:10.1017/S1464793106007007
West, G. B., J. H. Brown, and B. J. Enquist. 1997. A general model for the origin of allometric scaling laws in biology. Science 276: 122-126. doi:10.1126/science.276.5309.122

West, G. B., J. H. Brown, and B. J. Enquist. 1999a. A general model for the structure and allometry of plant vascular systems. Nature 400: 664-667. doi:10.1038/23251

West, G. B., J. H. Brown, and B. J. Enquist. 1999b. The fourth dimension of life: Fractal geometry and allometric scaling of organisms. Science 284: 1677-1679. doi:10.1126/ science.284.5420.1677

Yvon-Durocher, G., and others. 2012. Reconciling the temperature dependence of respiration across timescales and ecosystem types. Nature 487: 472-476. doi:10.1038/ nature11205

Zar, J. H. 1999. Biostatistical analysis, 4th ed. Prentice Hall.

\section{Acknowledgments}

CTK was supported by an NSF GRFP fellowship and NSF PRFB 1402074. Additional support came from NSF grants DEB-0845932 to EL and OCE-0928819 to EL and C Klausmeier. We appreciate the comments of two reviewers and K Edwards' assistance with biovolume data. This is W. K. Kellogg Biological Station contribution number 1919.

\section{Conflict of Interest}

None declared.

Submitted 09 August 2016 Revised 24 October 2016

Accepted 04 January 2017

Associate editor: Mikhail Zubkov 